\title{
ESTIMACIÓN DE LA RIQUEZA DE ESPECIES DE LA FAMILIA ASTERACEAE EN CUATRO ÁREAS PRIORITARIAS PARA LA CONSERVACIÓN DEL CENTRO DE MÉXICO UTILIZANDO MÉTODOS NO PARAMÉTRICOS PARA MEDIR LA BIODIVERSIDAD
}

\section{SPECIES RICHNESS ESTIMATION OF THE ASTERACEAE FAMILY IN FOUR AREAS FOR CONSERVATION FROM CENTRAL MEXICO USING NON-PARAMETRIC METHODS TO MEASURE BIODIVERSITY}

\author{
๑Diego Leyva-Castañeda, @Jesús Sigala-Rodríguez, @Gilberto Ocampo*
}

Departamento de Biología, Universidad Autónoma de Aguascalientes, Aguascalientes, México.

*Autor de correspondencia: gilberto.ocampo@edu.uaa.mx

\begin{abstract}
Resumen
Antecedentes: la familia Asteraceae es el grupo con más especies de angiospermas a nivel mundial (entre 24,000 - 33,000). A pesar de su diversidad, no se cuenta con suficiente información sobre la riqueza y distribución del grupo para su conservación en el centro de México.

Hipótesis: el uso de métodos no paramétricos para medir la diversidad de Asteraceae permitirá obtener información para promover su conservación en algunas áreas del centro de México.

Especies de estudio: especies de la familia Asteraceae localizadas en el centro de México.

Sitio y años de estudio: cuatro áreas prioritarias para la conservación sobre todo del estado de Aguascalientes: Sierra Fría, Sierra del Laurel, Tepezalá-Asientos y Juan El Grande (2016-2019).

Métodos: se utilizaron los métodos no paramétricos ICE y Chao2 para estimar la riqueza; se obtuvo una curva de acumulación de especies. Asimismo, se elaboró un mapa de riqueza de especies en una cuadrícula de $5 \times 5 \mathrm{~km}$ para identificar cuadrantes con riqueza alta. Se calculó la $\beta$-diversidad para medir diferencias en composición, anidamiento y recambio de especies entre áreas.

Resultados: los estimadores ICE y Chao2 indican una sobreestimación de la riqueza observada. El grado de completitud del inventario florístico de la familia en las áreas es de $62 \%$ para ICE y de $64 \%$ para Chao2. La $\beta$-diversidad indica alto recambio y pérdida de especies entre áreas.

Conclusiones: la riqueza y $\beta$-diversidad obtenidas son resultado del patrón espacial agrupado de las especies, presencia de especies exclusivas por área y preferencias y propiedades ecológicas específicas del grupo.

Palabras clave: Aguascalientes, $\beta$-diversidad, Chao2, Curva de acumulación de especies, Estimador de cobertura basado en incidencia.
\end{abstract}

\section{Abstract}

Background: The Asteraceae is the family with the highest number of angiosperm species $(24,000$ - 33,000). Despite this diversity, information on richness and distribution of the group for conservation purposes in central Mexico is scarce.

Hypothesis: The use of non-parametric methods to measure Asteraceae species diversity will allow to obtain information to promote the conservation of some areas in central Mexico.

Studied species: Species of the Asteraceae in four priority conservation areas in central Mexico.

Study area and dates: Four priority conservation areas mainly located in the state of Aguascalientes: Sierra Fría, Sierra del Laurel, TepezaláAsientos, and Juan El Grande (2016 to 2019).

Methods: Non-parametric methods ICE and Chao2 to estimate species richness were employed and a species accumulation curve was obtained. A species richness map was made in a $5 \times 5 \mathrm{~km}$ grid to identify quadrants with high species richness. $\beta$-diversity was calculated to measure differences in composition, nesting, and species turnover among areas.

Results: ICE and Chao2 estimators indicate an overestimation of the observed species richness. The degree of completeness of the floristic inventory for Asteraceae in the study areas is $62 \%$ for ICE and $64 \%$ for Chao2. $\beta$-diversity indicates high turnover and species lost among areas.

Conclusions: Richness and $\beta$-diversity obtained are the result of the species grouping spatial pattern, presence of exclusive species by area, and the ecological properties and preferences of the group.

Key words: Aguascalientes, $\beta$-diversity, Chao2, Incidence-based coverage estimator, Species accumulation curve. 
La familia Asteraceae (Compositae) es el grupo de plantas más diverso dentro de las angiospermas e incluye aproximadamente de 24,000 a 33,000 especies a nivel mundial (Funk et al. 2009, Mandel et al. 2017, RedondaMartínez 2018). México cuenta con alrededor de 314 a 387 géneros y 3,057 especies, de las cuales $66 \%$ son endémicas del país (Villaseñor et al. 1998, Villaseñor 2016). Las especies de la familia Asteraceae presentan una gran diversidad de formas de crecimiento, variabilidad morfológica, distribución geográfica y diversidad taxonómica (Villaseñor 2018). Por el gran número de especies y adaptaciones a distintos ambientes, también cumplen un papel destacado en estructura, composición y funcionamiento de las comunidades y ecosistemas (MazaVillalobos et al. 2014, Cuyckens et al. 2015). Muchas especies son pioneras en sucesiones primarias (e.g., especies de los géneros Acmella, Baccharis, Roldana y Verbesina (Martínez-Arévalo 2014, Olascuaga-Vargas et al. 2015), facilitando el establecimiento de otras especies al promover la maduración de los suelos (Katinas et al. 2007).

La familia Asteraceae tiene mecanismos muy eficientes para llevar a cabo dispersión anemócora (dispersión por viento) o zoocora (dispersión por animales) gracias al vilano presente en cipselas, presentan estrategias de reproducción sexual y asexual, tienen una alta capacidad adaptativa y se ven altamente favorecidas en eventos de sucesión ecológica (Sobrino 2012, Velásquez-Puentes \& Bacon 2016). Éstas son algunas características clave para el éxito de la preservación de la familia a nivel mundial, permitiéndole alcanzar una importante y vasta cobertura vegetal, incrementando el contenido de materia orgánica del ambiente y ayudando a la retención y estabilidad de los suelos (Villaseñor et al. 1998, Katinas et al. 2007, Del Vitto \& Petenatti 2009). La familia Asteraceae, debido a su alta y significativa representatividad dentro de la riqueza florística en diferentes regiones del país, es considerada un buen subrogado de la biodiversidad (Villaseñor et al. 2005a, 2007, Suárez-Mota et al. 2017, 2018, Villaseñor 2018); mientras que su alta riqueza en diversos sitios la hace un importante indicador de biodiversidad para establecer áreas prioritarias de conservación (Villaseñor et al. 1998, 2005b, Suárez-Mota et al. 2017, 2018).

La riqueza de especies es por lo común utilizada como herramienta para determinar la relevancia de un área en términos de biodiversidad y así poder delimitar las zonas prioritarias para la conservación; no obstante, el uso de la riqueza de especies para la toma de decisiones puede llegar a ser impreciso si no se tiene un listado completo de las especies presentes (Basualdo 2011). Los métodos no paramétricos son un conjunto de estimadores que no asumen un tipo de distribución estadística del conjunto de datos y no los ajustan a un modelo determinado (Moreno 2001). Estos métodos se enfocan en las especies poco abundantes o raras, es decir, las que se presentan en una o dos muestras y únicamente requieren datos de ausenciapresencia (López-Gómez \& Williams-Linera 2006). Algunas ventajas del uso de este tipo de estimadores son que pueden indicar qué tan completos están los inventarios, al tiempo que proporcionan una estimación del esfuerzo de muestreo necesario para tener un listado representativo y más completo de la biodiversidad en un área determinada (Basualdo 2011). De igual forma, pueden presentar un sesgo menor que la extrapolación basada en curvas de acumulación de especies y requieren de una cantidad menor de datos que los estimadores paramétricos (López-Gómez \& Williams-Linera 2006).

Los estimadores no paramétricos se han utilizado con la familia Asteraceae en México para evaluar qué tan completo es el catálogo nacional y cuánto esfuerzo de colecta falta por realizar; además, estos métodos han permitido comparar los valores de riqueza entre regiones, identificando así patrones de concentración de diversidad de esta familia para establecer programas adecuados de conservación y aprovechamiento de los recursos disponibles de áreas prioritarias (e.g., Villaseñor et al. 2005a, 2005b, 2007, García-Sánchez et al. 2014, Suárez-Mota et al. 2017, 2018). Por lo anterior, el objetivo del presente trabajo fue analizar la diversidad de la familia Asteraceae en cuatro áreas prioritarias de conservación en el estado mexicano de Aguascalientes, empleando métodos no paramétricos -con base en la riqueza de especies obtenida- para identificar sitios de particular importancia a los que se les debe dar una mayor prioridad en materia de conservación y que deben de ser considerados para futuras prospecciones florísticas.

\section{Materiales y métodos}

Se obtuvieron los polígonos correspondientes a las áreas prioritarias de conservación de la biodiversidad en Aguascalientes: Sierra Fría (SF), Sierra del Laurel (SL), Tepezalá-Asientos (TA) y Juan El Grande (LL) (Figura 1). El polígono para el área de SL (Aguascalientes, Jalisco y Zacatecas) fue basado en información del Gobierno del Estado de Aguascalientes (inédito). El polígono correspondiente a SF (Aguascalientes y Zacatecas) fue obtenido de CONABIO (2004). Los polígonos de las áreas de TA y de LL se obtuvieron a partir de CONABIO (2007).

A partir de 581 registros que incluyen 104 géneros y 224 especies de la familia Asteraceae se generó una base de datos. Los registros fueron obtenidos durante la revisión de material herborizado y usando las bases de datos de los herbarios EBUM, GUADA, HUAA, IEB, INEGI y de la Universidad Autónoma de Zacatecas (este último sin acrónimo registrado en el Index Herbariorum (http:// sweetgum.nybg.org/science/ih/), así como a partir de la información disponible en el portal web de la Global 
Biodiversity Information Facility (GBIF - www.gbif.org/). Los registros obtenidos fueron incluidos en un sistema de información geográfica (ArcGIS v. 10.5, ESRI 2011) para ubicar su presencia dentro de estas áreas y determinar el tipo de vegetación en que fueron colectados. Para los registros con los que no se contaba con georreferencia, se obtuvieron las coordenadas correspondientes de acuerdo con el programa GoogleEarth Pro a partir de los datos de las etiquetas de colecta. Los tipos de vegetación presentes en las áreas prioritarias de conservación se obtuvieron del catálogo de metadatos geográficos de uso de suelo y vegetación del INEGI agrupado por la CONABIO (2007).

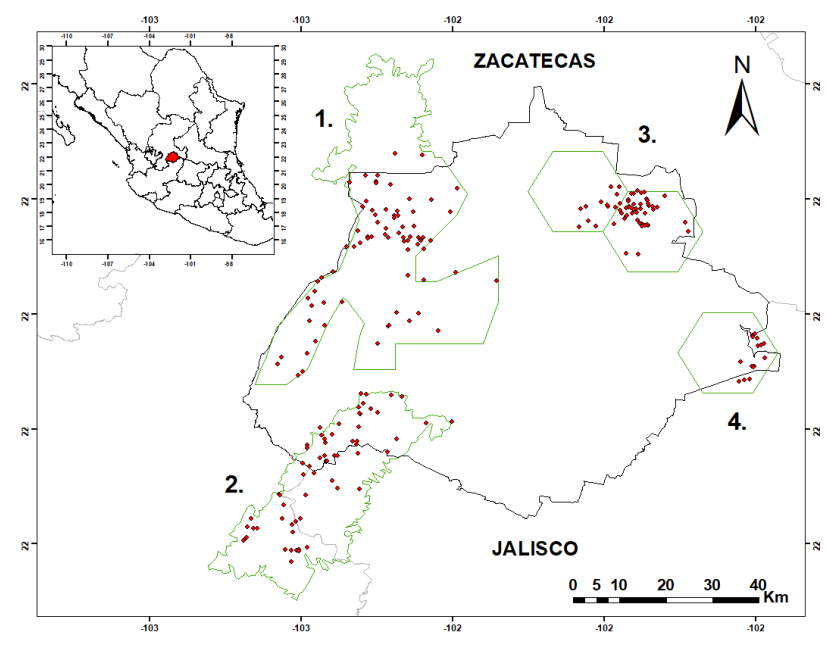

Figura 1. Mapa de Aguascalientes, México, donde se indican las cuatro áreas prioritarias para la conservación contempladas en el estudio (polígonos en color verde): 1. Sierra Fría, 2. Sierra del Laurel, 3. Tepezalá-Asientos y 4. Juan El Grande.

Para los cuatro polígonos bajo estudio se tienen registrados un total de nueve tipos de vegetación (Tabla 1); no obstante, cada tipo de vegetación dentro de cada uno de los polígonos se consideró como un sitio de colecta independiente. Por ejemplo, en tres de las cuatro áreas se encontró que el tipo de vegetación corresponde a bosque de encino (BE), por lo que se cuenta con tres unidades de muestreo independientes correspondientes al BE (SF-BE, SL-BE y LL-BE; Tabla 1). De esta manera, considerando todos los tipos de vegetación existentes en los polígonos estudiados, se tiene un total de 19 unidades de muestreo. Lo anterior se hizo con el fin de evaluar diferencias en riqueza de especies según el tipo de vegetación. Los sitios de muestreo se tomaron como valor de esfuerzo de colecta para la elaboración de una curva de acumulación de especies.

Para estimar los valores de riqueza para cada sitio, se

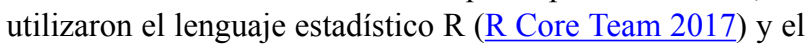
paquete estadístico BiodiversityR (Kindt \& Coe 2005). Para obtener el número de especies no observadas y la riqueza de especies por área con los índices no paramétricos basados en ICE (Incidence Coverage-based Estimator) y Chao2, se utilizó el paquete estadístico fossil (Vavrek 2015). Las curvas de acumulación de especies se elaboraron a partir de los cálculos de índices de biodiversidad obtenidos, graficando el número de especies observado contra el número de sitios de colecta presentes en cada área como esfuerzo de colecta. La riqueza de especies fue representada en un mapa cuadriculado, a partir de una capa de puntos georreferenciados, utilizando el programa ArcGis V.10.5 (ESRI 2011) de la siguiente manera: 1) se generó una cuadrícula de $5 \times 5 \mathrm{~km}$ sobre las áreas prioritarias con las herramientas ArcToolbox/Data management tools/Feature class/Create Fishnet ; 2) se creó una capa nueva uniendo la información de los puntos georreferenciados con la cuadrícula utilizando la herramienta Joins and Relates/ Join..., en la que cada celda de la cuadrícula tiene un valor único correspondiente a la suma de todos los puntos que caen dentro de ella (opción sum) y 3) se cambió la simbología de la capa nueva en Layer Properties/ Symbology, en donde a la suma de puntos se le asigna un color para representar la riqueza total por celda.

La beta diversidad o el recambio de especies entre comunidades fue calculada de acuerdo con el método propuesto por Baselga (2010, 2012) y Baselga \& Leprieur

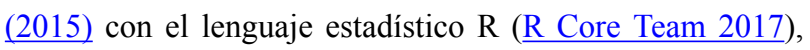
con el paquete betapart (Baselga et al. 2018). Baselga (2010) describe un método de estimación que separa la beta diversidad en dos componentes independientes: 1) la diferenciación en la composición de especies o recambio y 2) el anidamiento, comprendiendo valores entre un rango de 0 a 1 para ambos componentes, en donde valores cercanos a 1 indican que las comunidades presentan una baja similitud en la composición de especies. La diversidad beta se midió con los índices de disimilitud de Sorensen ( $\beta$ sor) y Jaccard $(\beta \mathrm{jac})$ de uso común para medir las diferencias en riqueza de especies entre comunidades (Calderón-Patrón et al. 2012). Para la diferenciación en la composición de especies entre comunidades, se utilizaron el índice de Simpson ( $\beta$ sim) y un componente de recambio del índice de Jaccard ( $\beta$ jtu) (Baselga 2010, 2012, Baselga \& Leprieur 2015, Calderón-Patrón et al. 2012); el anidamiento resultante se estimó con los componentes de disimilitud de Sorensen ( $\beta$ sne) y de Jaccard ( $\beta$ jne) (Baselga \& Leprieur 2015).

El cálculo de los componentes de beta diversidad (recambio y anidamiento) para definir, analizar y describir patrones espaciales de las comunidades puede ser difícil de interpretar; por ello, utilizar un método que integre todas estas aproximaciones resulta útil. Con las métricas antes mencionadas, se elaboró un gráfico de terna o piramidal en el software estadístico R con el paquete ggtern (Hamilton \& Ferry 2018) para describir el comportamiento de la riqueza 
Tabla 1. Tipos de vegetación según INEGI y agrupados por CONABIO (1998) presentes dentro de las cuatro áreas prioritarias para la conservación consideradas para el estudio.

\begin{tabular}{|c|c|c|c|c|}
\hline Tipo de vegetación & Sierra Fría (SF) & Sierra del Laurel (SL) & Tepezalá-Asientos (TA) & Juan El Grande (LL) \\
\hline Agricultura de riego (AR) & & $\mathrm{x}$ & $\mathrm{x}$ & \\
\hline Agricultura de temporal (AT) & & $\mathrm{x}$ & $\mathrm{x}$ & \\
\hline Bosque de encino (BE) & $\mathrm{x}$ & $\mathrm{x}$ & & $\mathrm{x}$ \\
\hline Matorral crasicaule (MC) & & & $\mathrm{x}$ & $\mathrm{x}$ \\
\hline Matorral subtropical (MS) & $\mathrm{x}$ & $\mathrm{x}$ & & \\
\hline Pastizal cultivado (PC) & $\mathrm{x}$ & $\mathrm{x}$ & $\mathrm{x}$ & \\
\hline Pastizal-Huizachal (PH) & & $\mathrm{x}$ & & \\
\hline Pastizal natural (PN) & $\mathrm{x}$ & & $\mathrm{x}$ & $\mathrm{x}$ \\
\hline Selva mediana subperennifolia (SMS) & & $\mathrm{x}$ & & \\
\hline
\end{tabular}

de especies por comunidad con respecto a un sistema de tres variables (e.g., $\beta$ sor, $\beta$ sne y $\beta$ sim) y visualizar qué patrones espaciales (agrupamiento o recambio) se presentan entre áreas. Se comparan las especies en común entre un par aleatorio de comunidades (representado por $\beta$ sne y $\beta \mathrm{jne}$ ), la ganancia de especies entre dos comunidades (dado por $\beta$ sim y $\beta j \mathrm{ju})$ y la pérdida de especies entre dos comunidades (dado por $\beta$ sor y $\beta j a c)$ (Koleff et al. 2003, Podani \& Schmera 2011).

\section{Resultados}

Para las cuatro áreas prioritarias contempladas en el estudio se encontraron un total de 224 especies observadas (Material suplementario 1); para ICE se estiman 368 especies y para Chao2 354 taxones (Tabla 2). El tipo de vegetación que contiene una mayor riqueza de especies es la de BE (139 especies), mientras que el PH presenta la riqueza más baja (una especie) (Tabla 3 ). En la Figura 3 se puede identificar dentro de las cuatro áreas de estudio cuáles son los sitios o celdas que presentan una mayor riqueza de especies. Destacan como celdas que requieren mayor esfuerzo de colecta 123 de ellas, que representan $66 \%$ del total $\left(615 \mathrm{~km}^{2}\right)$; siete celdas (4\% del total) contienen una riqueza de entre 21 a 56 especies, de las cuales solamente dos presentan un esfuerzo de colecta satisfactorio (TA - $77 \%$ y LL - $72 \%$ ). Para SF, 59 de 83 celdas requieren un mayor esfuerzo y faltan por colectar 45-57 especies, según los estimadores ICE y Chao2 respectivamente; para SL, 29 de 46 celdas requieren un esfuerzo adicional y faltan por colectar 128-198 especies; para TA, 22 de 34 celdas requieren esfuerzo adicional y faltan 25-27 especies; finalmente, para LL, 13 de 17 celdas requieren un esfuerzo adicional y faltan 22-27 especies por colectar.

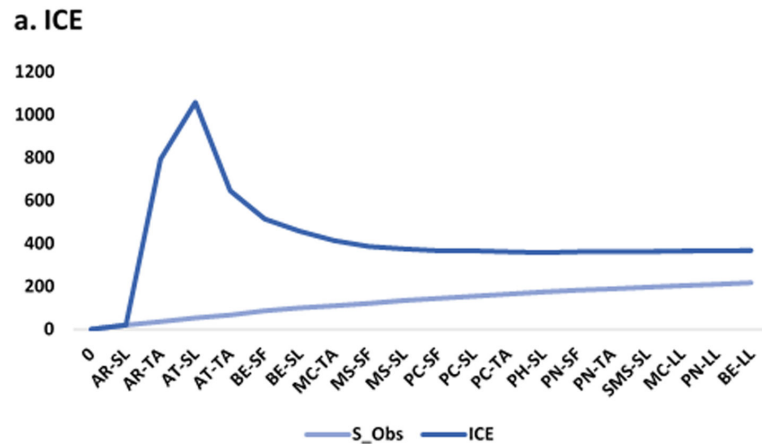

\section{b. Chao2}

600

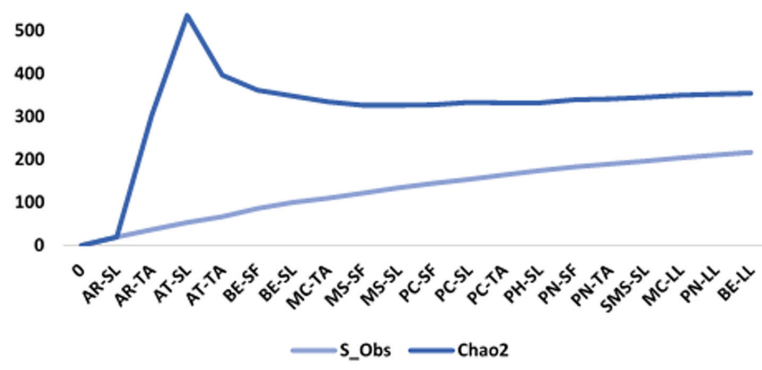

Figura 2. Curva de acumulación de especies de la familia Asteraceae de los polígonos bajo estudio de 1. Sierra Fría (SF), 2. Sierra del Laurel (SL), 3. Tepezalá-Asientos (TA) y 4. Juan El Grande (LL) utilizando los métodos no paramétricos ICE y Chao2. Se grafica el número de especies observadas (S_Obs) en las áreas de estudio contra el esfuerzo de colecta, siendo los tipos de vegetación presentes en donde se colectó (Tabla 1).

Para el cálculo de beta diversidad, recambio y anidamiento de especies entre comunidades (Tabla 4), los resultados son analizados y tomados entre rangos que van desde 0 a 1 . Mientras la beta diversidad ( $\beta$ sor y $\beta$ jac) se 
Tabla 2. Datos de riqueza total de especies de la familia Asteraceae y para cada una de las áreas prioritarias de conservación. Se muestran el número de especies observado y el número de especies estimado según los índices de Chao2 e ICE calculados en el ambiente estadístico R utilizando el paquete BiodiversityR ( $\underline{\text { Kindt \& Coe 2005). }}$.

\begin{tabular}{|c|c|c|c|c|c|c|}
\hline Riqueza de especies & & Cuatro áreas & Sierra Fría & Sierra del Laurel & Tepezalá-Asientos & Juan EI Grande \\
\hline Especies observadas & Total & 224 & 116 & 119 & 73 & 29 \\
\hline \multirow[t]{2}{*}{ Especies estimadas } & ICE & 368 & 161 & 317 & 98 & 56 \\
\hline & Chao2 & 354 & 173 & 247 & 100 & 51 \\
\hline Especies exclusivas & & & 54 & 56 & 29 & 5 \\
\hline Registros & & & 221 & 168 & 145 & 47 \\
\hline Singletons & & & 61 & 83 & 35 & 19 \\
\hline Doubletons & & & 32 & 27 & 22 & 9 \\
\hline
\end{tabular}

aproxime a 1, la composición de especies de una comunidad será marcadamente más distinta a las demás (Moreno 2001, Koleff et al. 2003, Baselga 2010). Para el caso de los polígonos de SF, SL, TA y LL, los valores estimados de $\beta$ sor y $\beta$ jac indican que estas áreas difieren ampliamente en la composición de especies, valores explicados por el alto recambio de especies entre estas comunidades; este es el patrón espacial principal que explica los valores de beta diversidad en las áreas de estudio, además de los bajos valores de anidamiento obtenidos.

De igual manera, se representó de manera gráfica la relación entre la riqueza de especies de cada sitio y tres variables distintas mediante un gráfico de terna (Figura 4), en donde $\boldsymbol{a}$ es el número total de especies que ocurren en dos cuadrantes; $\boldsymbol{b}$ es el número total de especies que ocurren en un sitio, pero no el de interés y $c$ es el número total de especies que ocurren en un sitio de interés, pero no se encuentran en un sitio vecino (Koleff et al. 2003). De manera general, 1) ßsne y ßjne describen cuáles son las especies en común entre un par de sitios o comunidades; 2 ) $\beta$ sim y $\beta$ jtu representan la ganancia de especies de un sitio con respecto a otro y, 3) $\beta$ sor y $\beta$ jac muestran las especies perdidas en un sitio con respecto a otro (Koleff et al. 2003, Podani \& Schmera 2011). En la Figura 4 se puede observar que las especies en común entre pares de comunidades son bajas por la posición basal de los puntos en el gráfico y hay sitios que presentan especies exclusivas o que sólo pueden ser encontradas en esa determinada comunidad con una tendencia a la pérdida de especies. Este patrón es observado sobre todo en las comunidades TA-LL y SL-LL, sitios con una composición de especies similar con respecto a las demás áreas, especies de distribución restringida a estas áreas y una tendencia a la pérdida de especies.

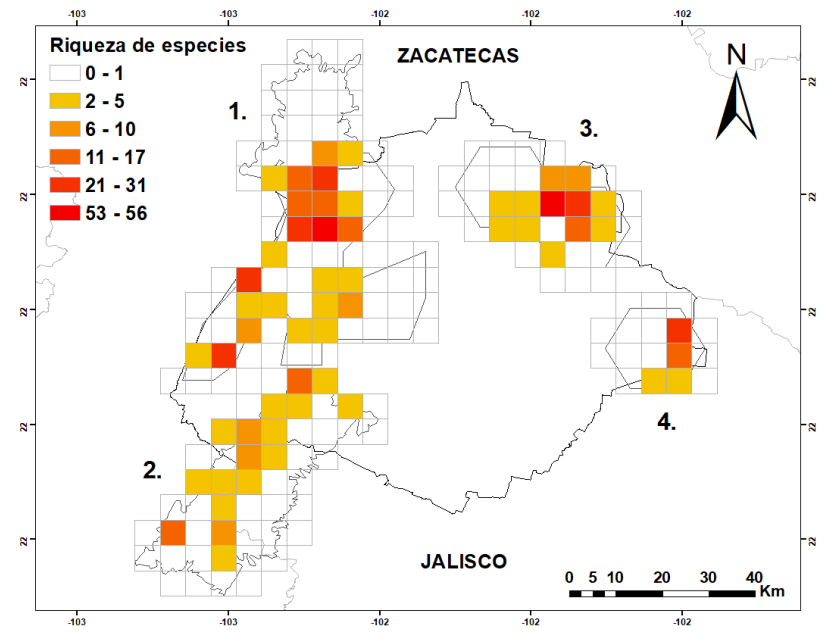

Figura 3. Mapa de riqueza de especies de la familia Asteraceae en 1) Sierra Fría, 2) Sierra del Laurel, 3) Tepezalá-Asientos y 4) Juan El Grande. Cada área está dividida por cuadrículas de $5 \times 5 \mathrm{~km}$ elaboradas en el programa ArcGis (ESRI 2011).

\section{Discusión}

Los estimadores no paramétricos ICE y Chao2, al contrario de los métodos paramétricos, no asumen el tipo de distribución estadística del conjunto de datos y no los ajustan a un modelo determinado, cuentan con un margen de error reducido y requieren una menor cantidad de información, pues sólo necesitan datos de incidencia o ausencia-presencia para su cálculo (Moreno 2001, Escalante 2003, López-Gómez \& Williams-Linera 2006, Chao \& Chiu 2016). Un buen estimador no paramétrico debe ser insensible al orden de las unidades de muestreo y al tamaño de la muestra, es decir, en una curva de acumulación de especies debe tener una tasa de crecimiento inicial alta hasta llegar a una riqueza máxima (Chazdon et al. 1998, LópezGómez \& Williams-Linera 2006, González-Oreja et al. 
Asteraceae en cuatro áreas prioritarias para la conservación

Tabla 3. Riqueza total de especies de la familia Asteraceae en cada tipo de vegetación y por sitio de colecta.

\begin{tabular}{|c|c|c|c|}
\hline Tipo de vegetación & Riqueza de especies & Sitio de colecta & Riqueza de especies \\
\hline \multirow[t]{2}{*}{ Agricultura de riego } & 27 & SL & 19 \\
\hline & & TA & 8 \\
\hline \multirow[t]{2}{*}{ Agricultura de temporal } & 45 & SL & 6 \\
\hline & & $\mathrm{TA}$ & 41 \\
\hline \multirow[t]{3}{*}{ Bosque de encino } & 139 & SF & 116 \\
\hline & & SL & 35 \\
\hline & & LL & 3 \\
\hline \multirow[t]{2}{*}{ Matorral crasicaule } & 9 & TA & 1 \\
\hline & & LL & 8 \\
\hline \multirow[t]{2}{*}{ Matorral subtropical } & 46 & $\mathrm{SF}$ & 29 \\
\hline & & SL & 19 \\
\hline \multirow[t]{3}{*}{ Pastizal cultivado } & 54 & $\mathrm{SF}$ & 23 \\
\hline & & SL & 6 \\
\hline & & TA & 30 \\
\hline \multirow[t]{3}{*}{ Pastizal natural } & 37 & $\mathrm{SF}$ & 11 \\
\hline & & $\mathrm{TA}$ & 10 \\
\hline & & LL & 23 \\
\hline Pastizal-huizachal & 1 & SL & 1 \\
\hline Selva mediana subperennifolia & 20 & SL & 20 \\
\hline
\end{tabular}

2010). Las curvas de acumulación de especies obtenidas con los estimadores no paramétricos ICE y Chao2 tienen una tasa alta de crecimiento inicial, pero se encuentran muy por encima de los valores de riqueza de especies observadas, lo que indica una sobreestimación de la riqueza de especies observadas (Figura 2). El error de sobreestimación de la riqueza en estas comunidades indica que presentan una distribución espacial agregada debido a las preferencias ecológicas o geográficas del grupo de estudio, es decir, especies de la familia Asteraceae presentan una tendencia a concentrarse en partes específicas del hábitat debido a las condiciones heterogéneas del área como lo son: el relieve, la exposición a viento y la humedad, el uso de suelo y el impacto antropogénico, entre otras (Moreno 2001, De Mas-Castroverde 2007, Villaseñor 2010, Pérez-Paredes et al. 2014, Hernández et al. 2017). El $\mathrm{BE}$ del área de SF es el sitio donde se concentran más especies, siendo este el tipo de vegetación de mayor cobertura entre las cuatro áreas de estudio (CONABIO 1998, 2004, CONABIO 2007, Ávila-Villegas et al. 2008) y donde se ven más beneficiadas especies de este grupo, ya que la familia Asteraceae alcanza una mayor diversidad en las zonas templadas (e.g., BE o encino-pino) y tiende a disminuir en zonas tropicales y húmedas (Tapia-Muñoz 2010, García-Sánchez et al. 2014, Villaseñor 2018,
Carrasco-Ortiz et al. 2019). Los pastizales cultivados le siguen al BE en riqueza de especies (Siqueiros-Delgado et al. 2016, Villaseñor 2018), ya que los medios ruderales y arvenses en ambientes secos y templados son áreas idóneas para el desarrollo de especies de la familia Asteraceae, llegando a representar en algunos casos una seria amenaza para cultivos y otras especies silvestres (Tapia-Muñoz 2010). El tercer sitio que presenta una riqueza alta de especies es el matorral subtropical, un tipo de vegetación ruderal o secundaria, en donde las especies de compuestas asociadas a disturbio se ven altamente favorecidas (TapiaMuñoz 2010, Zamora-Crescencio et al. 2011, Rzedowski et al. 2014).

Tabla 4. Cálculo de $\beta$-diversidad, anidamiento y el recambio de especies entre áreas prioritarias utilizando el método de Baselga

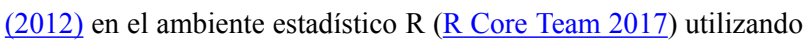
el paquete betapart (Baselga et al. 2018).

\begin{tabular}{|c|c|c|c|}
\hline$\beta$-diversidad & Anid & Imiento & Recambio \\
\hline ßjac 0.8581 & $\beta$ jne & 0.082 & Bjtu 0.7756 \\
\hline Bsor 0.7515 & Bsne & 0.118 & Bsim 0.6335 \\
\hline
\end{tabular}


a. Jaccard

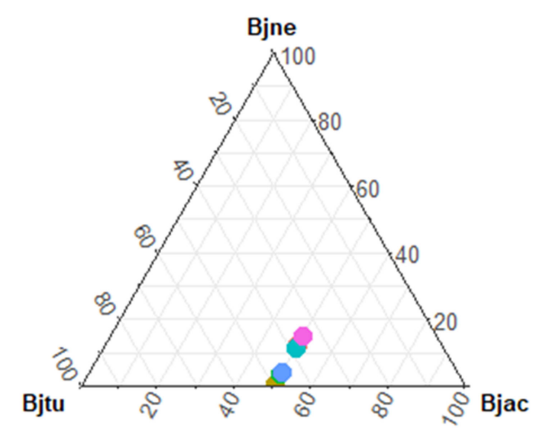

b. Sorensen

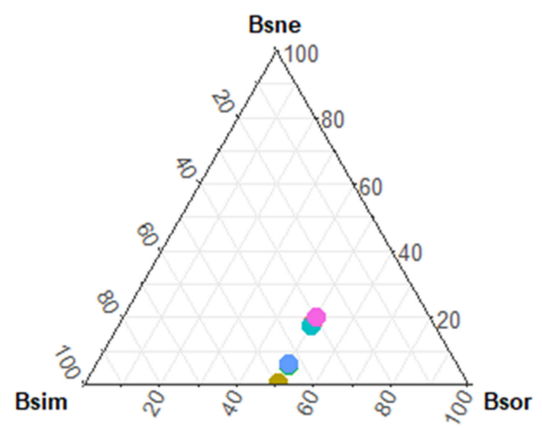

Figura 4. Gráficas de terna que comparan los valores de beta diversidad (Sorensen y Jaccard) entre pares de áreas (Tabla 1). Se comparan la composición ( $\beta$ jne y $\beta$ sne), la pérdida ( $\beta$ jtu y $\beta \operatorname{sim}$ ) y la ganancia de especies ( $\beta$ jac y $\beta$ sor) entre pares de áreas.

La diferencia entre los valores de especies observadas y estimadas también puede explicarse por otros factores que modifican el comportamiento y la precisión de estos estimadores: 1) el número de recolectores involucrados, siendo por lo general de dos a tres por salida para abarcar la mayor extensión de área posible por punto de colecta o bien registros colectados por grupos de estudio durante años anteriores; 2) los periodos de recolecta, con salidas una vez por semana a lo largo de todo el trabajo de investigación o salidas realizadas una vez por semestre por parte de grupos de estudio y 3) la cercanía de las áreas a asentamientos o poblados, procurando colectar en sitios lo más alejados y lo menos impactados posibles (Moreno 2001). Los factores mencionados también influyen de manera determinante en la concentración de colectas hacia ciertos sitios de manera preferente. Lo anterior hace que el muestreo no haya sido homogéneo entre las localidades, requiriendo de un número más grande de unidades de muestreo para completar el inventario florístico y obtener valores de ICE y Chao2 más cercanos a la realidad (Chazdon et al. 1998, López-Gómez $\underline{\text { \& Williams-Linera 2006, Villaseñor et al. 2013, Chao \& }}$ Chiu 2016).
Un ejemplo de lo anterior se observa en el polígono de TA (Figura 3), en donde el cerro de Altamira concentra una gran cantidad de registros con respecto a los demás cuadrantes dentro de esta área, así como en el polígono LL, que tiene la mayor parte de los registros concentrados en la principal serranía que lleva el mismo nombre. En parte, este fenómeno puede deberse a que un área considerable de estos polígonos se encuentra impactada sustancialmente por actividades humanas, como terrenos de cultivo (de temporal y de riego), presencia de asentamientos humanos, terracerías y carreteras en donde la colecta botánica no es frecuente. Los polígonos de SF y SL presentan un menor impacto por estos factores, por lo que los tipos de vegetación natural en esta zona se encuentran mejor conservados, ayudando a que las colectas sean más frecuentes y estén mejor distribuidas. Lo anterior se aprecia de mejor manera en el mapa de riqueza de especies (Figura 3). De manera general, el esfuerzo de colecta no es satisfactorio, pues se observan varias celdas en blanco que requieren un muestreo sustancial para poder documentar de una mejor manera la riqueza de la familia Asteraceae en estas áreas prioritarias.

Villaseñor et al. (2005b) mencionan que el error de sobreestimación y los valores obtenidos con índices no paramétricos, como los utilizados en este trabajo, son producto de una falta de repeticiones en el esfuerzo de colecta y la falta de heterogeneidad. Esta última es originada por el tamaño de las celdas, ya que mientras más grande sea el área existe la posibilidad de que los muestreos reflejen la heterogeneidad de las áreas; mientras más se reduzca el tamaño de las celdas, aumenta la probabilidad de muestreos únicos y disminuye la de tener un muestreo con más especies infrecuentes. También es de considerar que el origen de una cuadrícula para cualquier mapa es arbitrario y elaborado conforme a la extensión geográfica con la que se esté trabajando o según las necesidades del investigador, lo cual puede influir directamente en los resultados obtenidos (Vargas-Amado et al. 2013).

Estudios sobre los factores que originan patrones de beta diversidad mencionan que ésta incrementa por la variación ambiental entre ecosistemas y en latitudes menores, propiciando una riqueza alta y recambio de especies en áreas tropicales (Koleff et al. 2003). Lo anterior se origina a través de eventos de migración, especiación y aislamiento de ecosistemas durante periodos de glaciación (Baselga 2010). Estas afirmaciones al ser probadas en otros trabajos resultan ser inconsistentes, pues valores de beta diversidad y de recambio de especies son independientes al tipo de ambiente o hábitat (Koleff et al. 2003). Se proponen entonces otros mecanismos que expliquen la beta diversidad de las comunidades entre los cuales se encuentran: 1) la distancia entre sitios o comunidades con valores de bajos a moderados entre sitios muy cercanos y valores altos de 
recambio entre sitios muy alejados (Harte \& Kinzing 1997, Koleff et al. 2003) (el valor obtenido de recambio de especies en los sitios estudiados no concuerda con el supuesto de distancias entre áreas, ya que a pesar de que éstas se encuentran muy próximas geográficamente, los valores de beta diversidad y de recambio son altos, lo que sugiere la presencia de otro mecanismo que explique los valores obtenidos de beta diversidad y de la pérdida de especies en estas áreas); 2) la presencia de especies endémicas o de distribución restringida de manera local, esto es, especies que se encuentran solamente en una de las cuatro áreas bajo estudio (en Aguascalientes se tiene registrada la presencia de 192 especies endémicas de México, pero ninguna endémica para el estado (Villaseñor 2018); además de las 224 especies consideradas en este estudio, 167 presentan una distribución restringida, siendo estas últimas las que aportan directamente al valor de recambio de un sitio con respecto a otro y diferencias entre composición de especies entre comunidades (Koleff et al. 2003) y 3) las características o propiedades ecológicas del taxón que se estudia, es decir, caracteres morfológicos, químicos o hábitos que influyen en la distribución de las especies (Calderón-Patrón et al. 2012). Como ya se mencionó, la familia Asteraceae tiene mecanismos muy eficientes para su dispersión y adaptación, características que le permite tener una amplia distribución favoreciendo bajos valores de recambio entre las comunidades (Gering \& Crist 2002, Koleff et al. 2003, Calderón-Patrón et al. 2012). Pese a lo anterior, los valores de recambio entre sitios son altos y la distribución espacial de las especies no es uniforme, sino agrupada o en parches.

Un $80 \%$ de la cubierta vegetal del estado ha sido modificada, mientras que $90 \%$ del suelo presenta una marcada erosión, originando una pérdida de la biodiversidad vegetal (Ávila-Villegas et al. 2008). La pérdida de especies en las áreas prioritarias se atribuye a diversos factores, entre los que se encuentran: 1) la localización del estado en la planicie central árida del país, 2) el tamaño pequeño del estado y 3) una topografía accesible (Siqueiros-Delgado et al. 2016). Lo anterior se incorpora a la presencia de una marcada actividad antropogénica en zonas pobladas, de cultivo, ganadería o minería. Estas actividades promueven la transformación, perturbación, degradación y fragmentación en las áreas prioritarias (Siqueiros-Delgado et al. 2016), además de que aportan a la diferencia en la composición y al recambio de especies, se registra que mientras mayor sea la diferencia en composición y más alto el valor obtenido de recambio se incrementará la pérdida de especies entre pares sitios (Koleff \& Gaston 2002, Koleff et al. 2003, Baselga 2010, Calderón-Patrón et al. 2012).

Los resultados de este análisis brindan una idea del camino que se debe tomar para proponer y reforzar las estrategias de conservación de la familia Asteraceae en el estado de Aguascalientes. A pesar de la presencia de cuadrantes con una diversidad baja, existen sitios que pueden servir como reservorios y áreas de conservación de especies de esta familia. Para SF y SL se debe prestar particular atención en sitios o cuadrantes dentro de los BE que presentaron la mayor riqueza de especies (Figura 3). Debido a la poca perturbación antrópica y preferencias ecológicas de la familia, el establecer reservas y áreas de trabajo para la conservación alrededor de estos sitios parece una estrategia factible. En cuanto a TA y LL, independientemente del tipo de vegetación y preferencias del grupo de estudio, las estrategias de conservación deben ser implementadas alrededor de las serranías de Altamira y Juan El Grande, las cuales presentaron valores de riqueza altos; dada la creciente actividad antrópica alrededor de estos sitios, los resultados indican una pérdida de la diversidad de Asteraceae en estas áreas, además de que la diversidad, las colectas y los registros disponibles se encuentran preferencialmente restringidas a estas serranías.

El nivel de completitud de la riqueza de especies de la familia Asteraceae en las áreas de estudio es de alrededor de $60 \%$ y para tener un listado florístico del grupo en la región lo más completo posible, el esfuerzo de colecta debe incrementarse; se propone así aplicar estrategias y metodologías eficientes de colecta en los cuadrantes que presentan una riqueza baja de especies. Se deben formular estrategias adecuadas de conservación en los sitios que presentaron los valores de riqueza más altos (SF y $\mathrm{SL}$ ), especialmente en los cuadrantes dentro de BE donde se concentra la mayor diversidad de compuestas. En TA y LL, las serranías principales de cada área representan sitios de interés para poder desarrollar estrategias de conservación.

Las diferencias entre la extensión geográfica de las áreas de estudio, la forma en que las especies se encuentran distribuidas y la presencia de perturbación, fragmentación y degradación del ambiente, son factores que afectan directamente en la estimación de los valores de riqueza. Ejemplo de lo anterior es la sobreestimación de la riqueza en las cuatro áreas y una beta diversidad alta explicada por valores de recambio altos y la pérdida de especies entre las comunidades. La familia Asteraceae es un importante componente que brinda estabilidad a los ecosistemas y provee una gran variedad de servicios ecológicos. Este estudio muestra una aproximación de la riqueza, distribución y ecología de comunidades vegetales de esta familia en Aguascalientes, aspectos que no habían sido estudiados o analizados para el estado ni para las áreas prioritarias para la conservación dentro de él. Dado que se muestra un gran número de especies que no son compartidas entre áreas y las especies presenten un tipo de distribución agrupada (ya sea por preferencias ecológicas o por actividad antrópica), se considera que se debe prestar particular atención y esfuerzo a los sitios donde se 
concentran la mayor cantidad de especies de esta familia, el tipo de vegetación y requerimientos ecológicos adecuados para el desarrollo y la subsistencia de esta familia para evitar la pérdida de especies entre comunidades.

\section{Agradecimientos}

El primer autor agradece al Consejo Nacional de Ciencia y Tecnología (CONACYT) el apoyo económico para efectuar estudios de doctorado en la Universidad Autónoma de Aguascalientes. Este trabajo fue financiado por el CONACYT (proyecto CB-2015-01-256048) y la Universidad Autónoma de Aguascalientes (proyecto PIB17-7N). A Ricardo Rivera Martínez, Andrés Alejandro Mares Guerrero y Juan Carlos Díaz de León que apoyaron en la colecta de ejemplares durante las salidas a campo en las cuatro áreas de estudio y en la identificación de las especies en el herbario HUAA. A encargados y personal de herbarios y colecciones visitadas que brindaron su apoyo y dieron facilidades para las consultas realizadas.

\section{Material Suplementario}

El material suplementario de este artículo puede consultarse aquí: https://doi.org/10.17129/botsci.2552

\section{Literatura citada}

Ávila-Villegas H, Melgarejo D, Cruz-Aragón A, coord. 2008. La Biodiversidad en Aguascalientes. Estudio de Estado. Comisión Nacional para el Conocimiento y Uso de la Biodiversidad, Instituto del Medio Ambiente del estado de Aguascalientes, Universidad Autónoma de Aguascalientes. Aguascalientes, México. ISBN: 978-970-9000-45-0

Baselga A. 2010. Partitioning the turnover and nestedness components of beta diversity. Global Ecology \& Biogeography 19: 134-143. DOI: https://doi.org/10.1111/ j.1466-8238.2009.00490.x

Baselga A. 2012. The relationship between species replacement, dissimilarity derived from nestedness, and nestedness. Globlal Ecology \& Biogeography 21: 1223-1232. DOI: https://doi.org/10.1111/j.1466-8238. 2011.00756.x

Baselga A, Leprieur F. 2015. Comparing methods to separate components of beta diversity. Methods in Ecology and Evolution 6: 1069-1079. DOI: https:// doi.org/10.1111/2041-210X.12388

Baselga A, Orme C, Villéger S, De Bortoli J, Leprieur F. 2018. betapart: Partitioning beta diversity into turnover and nestedness components. $\mathrm{R}$ package version 1.5.1. http://CRAN.R-project.org/package=betapart (accessed June 1, 2019).
Basualdo CV. 2011. Choosing the best non-parametric richness estimator for benthic macroinvertebrates databases. Revista de la Sociedad Entomológica Argentina 70: 27-38.

Calderón-Patrón JM, Moreno CE, Zuria I. 2012. La diversidad beta: medio siglo de avances. Revista Mexicana de Biodiversidad 83: 879-891. DOI: https:// doi.org/10.7550/rmb.25510

Carrasco-Ortiz M, Munguía-Lino G, Castro-Castro A, Vargas-Amado G, Harker M, Rodríguez A. 2019. Riqueza, distribución geográfica y estado de conservación del género Dahlia (Asteraceae) en México. Acta Botanica Mexicana 126: 1-24. DOI: https://doi.org/ $\underline{10.21829 / \mathrm{abm} 126.2019 .1354}$

Chao A, Chiu CH. 2016. Species richness: estimation and comparison. Wiley StatsRef: Statistics Reference Online. 1-26. DOI: https://doi.org/10.1002/9781118445112.stat 03432.pub2

Chazdon RL, Colwell RK, Denslow JS y Guariguata MR. 1998. Statistical methods for estimating species richness of woody regeneration in primary and secondary rain forest of northeastern Costa Rica. In: Dallmeier F, Comiskey JA, eds. Forest Biodiversity Research, Monitoring and Modeling: Conceptual Background and Old World Case Studies. Paris: UNESCO, pp. 285-309. ISBN 10: 92-3-103408-1; ISBN-13: 978-9231034084

CONABIO [Comisión Nacional para el Conocimiento y Uso de la Biodiversidad]. 1998. Uso de suelo y vegetación de INEGI agrupado por CONABIO. Escala 1:1000000. Comisión Nacional para el Conocimiento y Uso de la Biodiversidad. México. http://www. conabio.gob.mx/informacion/gis/maps/geo/ usva1mgw.zip (accessed September 23, 2019).

CONABIO. 2004. Regiones Terrestres Prioritarias. Escala 1:1000000. Comisión Nacional para el Conocimiento y Uso de la Biodiversidad. México. http://www. conabio.gob.mx/conocimiento/regionalizacion/doctos/ Tlistado.html (accessed September 23, 2019).

CONABIO. 2007. Sitios prioritarios terrestres para la conservación de la biodiversidad. Escala 1: 1000000. México, DF: Comisión Nacional de Áreas Naturales Protegidas-The Nature Conservancy - Programa MéxicoPronatura. http://www.conabio.gob.mx/informacion/gis/ maps/geo/spt1mgw.zip (accessed September 23, 2019).

Cuyckens GE, Malizia LR, Blundo C. 2015. Composición, diversidad y estructura de comunidades de árboles en un gradiente altitudinal de selvas subtropicales de montaña (Serranías de Zapla, Jujuy, Argentina). Madera y Bosques 21: 137-148. DOI: https://doi.org/10.21829/ $\underline{\text { myb.2015.213463 }}$

De Mas-Castroverde E. 2007. Evaluación y Predicción de la Biodiversidad. Un modelo con Araneidos en el Parque 
Natural del Cadi-Moixeró. PhD. Thesis. Universidad de Barcelona.

Del Vitto LA, Petenatti EM. 2009. Asteráceas de importancia económica y ambiental. Primera parte. Sinopsis Morfológica y taxonómica, importancia ecológica y plantas de interés industrial. Multequina 18: 87-115.

Escalante T. 2003. ¿Cuántas especies hay? Los estimadores no paramétricos de Chao. Elementos 52: 53-56.

ESRI [Environmental Systems Research Institute]. 2011. ArcGIS 10. Redlands California.

Funk VA, Susanna A, Stuessy TF, Bayer RJ. 2009. Systematics, Evolution, and Biogeography of Compositae. Vienna: International Association for Plant Taxonomy.

García-Sánchez CA, Sánchez-González A, Villaseñor JL. 2014. La familia Asteraceae en el parque nacional Los Mármoles, Hidalgo, México. Acta Botanica Mexicana 106: 97-116. DOI: https://doi.org/10.21829/abm106. 2014.219

Gering JC, Crist TO. 2002. The Alpha-beta-regional relationship: providing new insights into local-regional patterns of species richness and scale dependence of diversity components. Ecology Letters 5: 433-444. DOI: https://doi.org/10.1046/j.1461-0248.2002.00335.x

González-Oreja JA, Fuente-Díaz-Ordaz AA, HernándezSantín L, Buzo-Franco D, Bonache-Regidor C. 2010. Evaluación de estimadores no paramétricos de la riqueza de especies. Un ejemplo con aves en áreas verdes de la ciudad de Puebla, México. Animal Biodiversity and Conservation 33.1: 31-45.

Hamilton NE, Ferry M. 2018. ggtern: Ternary Diagrams Using ggplot2. Journal of Statistical Software, Code Snippets 87: 1-17. DOI: https://doi.org/10.18637/jss. $\underline{\mathrm{v} 087 . \mathrm{c} 03}$

Harte J, Kinzing P. 1997. On the implications of speciesarea relationships for endemism, spatial turnover, and food web patterns. Oikos 80: 417-427. DOI: https:// doi.org/10.2307/3546614

Hernández FJ, Navarro-Mata CB, Peña-Montañez R, Nájera-Luna A. 2017. Patrón de distribución espacial de las especies arbóreas de la región de El Salto, Durango. Revista Mexicana de Ciencias Forestales 9: 169-186. DOI: https://doi.org/10.29298/rmcf.v9i47.161

Katinas L, Gutiérrez DG, Grossi MA, Crisci JV. 2007. Panorama de la familia Asteraceae (=Compositae) en la República Argentina. Boletín de la Sociedad Argentina de Botánica 42: 113-129.

Kindt R, Coe R. 2005. Tree Diversity Analysis: A Manual and Software for Common Statistical Methods for Ecological and Biodiversity Studies. Nairobi: World Agroforestry Centre (ICRAF). ISBN 10: 92-9059-179-X; ISBN-13: 978-9290591795
Koleff P, Gaston KJ. 2002. The relationship between local and regional species richness and spatial turnover. Global Ecology \& Biogeography 11: 363-375. DOI: https://doi.org/10.1046/j.1466-822x.2002.00302.x

Koleff P, Gaston KJ, Lennon JJ. 2003. Measuring beta diversity for presence-absence data. Journal of Animal Ecology 72: 367-382. DOI: https://doi.org/10.1046/ j.1365-2656.2003.00710.x

López-Gómez AM, Williams-Linera G. 2006. Evaluación de métodos no paramétricos para la estimación de riqueza de especies de plantas leñosas en cafetales. Boletín de la Sociedad Botánica de México 78: 7-15. DOI: https://doi.org/10.17129/botsci.1717

Mandel JR, Barker MS, Bayer RJ, Dikow RB, Gao T, Jones KE, Keeley S, Kilian N, Ma H, Siniscalchi CM, Susanna A, Thapa R, Watson L, Funk VA. 2017. The compositae tree of life in the age of phylogenomics. Journal of Systematics and Evolution 55: 405-410. DOI: https:// doi.org/10.1111/jse. 12265

Martínez-Árevalo JV. 2014. Sucesión vegetal en bordes de bosques de Pinabete (Abies guatemalensis Rehder) del occidente de Guatemala. Revista Mexicana de Ciencias Forestales 5: 64-77. DOI: https://doi.org/10.29298/ rmcf.v5i23.342

Maza-Villalobos S, Macedo-Santana F, RodríguezVelázquez J, Oyama K, Martínez-Ramos M. 2014. Variación de la estructura y composición de comunidades de árboles y arbustos entre tipos de vegetación en la Cuenca de Cuitzeo, Michoacán. Botanical Sciences 92: 243-258. DOI: https://doi.org/ 10.17129/botsci.104

Moreno CE. 2001. Métodos para Medir la Biodiversidad. Zaragoza: Programa Iberoamericano de Ciencia y Tecnología para el Desarrollo - Oficina Regional de Ciencia y Tecnología para América Latina y el Caribe, UNESCO - Sociedad Entomológica Aragonesa. ISBN: 84-922495-2-8

Olascuaga-Vargas D, Mercado-Gómez J, Sánchez-Montaño LR. 2015. Análisis de la vegetación sucesional en un fragmento de bosque seco tropical en Toluviejo-Sucre (Colombia). Colombia Forestal 19: 23-40. DOI: https:// doi.org/10.14483/udistrital.jour.colomb.for.2016.1.a02

Pérez-Paredes MG, Sánchez-González A, Tejero-Diez JD. 2014. Estructura poblacional y características del hábitat de dos especies de Cyatheaceae del estado de Hidalgo. Botanical Sciences 92: 259-271. DOI: https://doi.org/ $\underline{10.17129 / \text { botsci.48 }}$

Podani J, Schmera D. 2011. A new conceptual and methodological framework for exploring and explaining pattern in presence-absence data. Oikos 120: 1625-1638. DOI: https://doi.org/10.1111/j.1600-0706.2011.19451.x 
R Core Team. 2007. R: a language and environment for statistical computing. $\mathrm{R}$ Foundation for Statistical Computing, Vienna, Austria. http://www.R-project.org

Redonda-Martínez R. 2018. Tratamiento taxonómico de la tribu Mutisieae (Asteraceae) en México. Acta Botanica Mexicana 123: 121-166. DOI: http://dx.doi.org/ $\underline{10.21829 / \mathrm{abm} 123.2018 .1277}$

Rzedowski J, Zamudio S, Calderón de Rzedowski G, Paizanni A. 2014. El bosque tropical caducifolio en la cuenca lacustre de Pátzcuaro (Michoacán, México. Flora del Bajío y Regiones Adyacentes (Fascículo Complementario) XXIX: 1-18.

Siqueiros-Delgado MA, Rodríguez-Ávalos JA, MartínezRamírez JM, Sierra-Muñoz JC. 2016. Situación actual de la vegetación del estado de Aguascalientes, México. Botanical Sciences 94: 455-470. DOI: https://doi.org/ $\underline{10.17129 / \text { botsci.466 }}$

Sobrino E. 2012. El control de las invasiones biológicas, un difícil reto ambiental. Foresta 56: 8-13.

Suárez-Mota ME, Villaseñor JL, López-Mata L. 2017. Dominios climáticos de la Sierra Madre Oriental y su relación con la diversidad florística. Revista Mexicana de Biodiversidad 88: 224-233. DOI: https://dx.doi.org/ 10.1016/j.rmb.2017.01.020

Suárez-Mota ME, Villaseñor JL, Ramírez-Aguirre MB. 2018. Sitios prioritarios para la conservación de la riqueza florística y el endemismo de la Sierra Norte de Oaxaca, México. Acta Botanica Mexicana 124: 1-29. DOI: $\underline{\text { https://doi.org/10.21829/abm124.2018.1296 }}$

Tapia-Muñoz JL. 2010. La familia Asteraceae. Desde el herbario CICY. 2: 82-84.

Vargas-Amado G, Castro-Castro A, Harker M, Villaseñor J L, Ortiz E, Rodríguez A. 2013. Distribución geográfica y riqueza del género Cosmos (Asteraceae: Coreopsidae). Revista Mexicana de Biodiversidad 84: 536-555. DOI: http://dx.doi.org/10.7550/rmb.31481

Vavrek MJ. 2015. Package "fossil". Palaeoecological and Palaeogeographical Analysis Tools. https://cran.rproject.org/web/packages/fossil/index.html (accessed June 1, 2019).

Velásquez-Puentes FJ, Bacon CD. 2016. Una introducción a la estructura filogenética de comunidades: Un caso de estudio en palmas de Bolivia. Ecología en Bolivia 51: 126-140.

Editor de sección: Monserrat Vázquez Sánchez

Contribución de los autores: DLC, trabajo de campo, compilación y análisis de datos, revisión de especímenes, diseño del modelo experimental; JSR, diseño del modelo experimental; GO, diseño del modelo experimental. Todos los autores contribuyeron a la discusión, revisión y aprobación del manuscrito final.
Villaseñor JL. 2010. El bosque húmedo de montaña en México y sus plantas vasculares: catálogo florísticotaxonómico. México, DF: Universidad Nacional Autónoma de México y Comisión Nacional para el Conocimiento y Uso de la Biodiversidad. ISBN: 978-607-02-1557-5

Villaseñor JL. 2016. Checklist of the native vascular plants of Mexico. Revista Mexicana de Biodiversidad 87: 559-902. DOI: http://dx.doi.org/10.1016/j.rmb.2016. 06.017

Villaseñor JL. 2018. Diversidad y distribución de la familia Asteraceae en México. Botanical Sciences 96: 332-358. DOI: http://dx.doi.org/10.17129/botsci.1872

Villaseñor JL, Ibarra G, Ocaña D. 1998. Strategies for the conservation of Asteraceae in Mexico. Conservation Biology 12: 1066-1075.

Villaseñor JL, Ibarra-Manríquez G, Meave JA, Ortiz E. 2005a. Higher taxa as surrogates of plant biodiversity in a megadiverse country. Conservation Biology 19: 232-238. DOI: https://doi.org/10.1111/j.1523-1739.2005. 00264.X

Villaseñor JL, Maeda P, Colín-López JJ, Ortiz E. 2005b. Estimación de la riqueza de especies de Asteraceae mediante extrapolación a partir de datos de presenciaausencia. Boletín de la Sociedad Botánica de México 76: 5-18. DOI: https://doi.org/10.17129/botsci.1700

Villaseñor JL, Maeda P, Rosell JA, Ortiz E. 2007. Plant families as predictors of plant biodiversity in Mexico. Diversity and Distributions 13: 871-876. DOI: https:// doi.org/10.1111/j.1472-4642.2007.00385.x

Villaseñor JL, Ortiz E, Cadena J, Estrada AS. 2013. Patrones de riqueza florística en el estado de Jalisco: La tribu Senecioneae (Asteraceae) como estudio de caso. Ibugana 4: 63-78.

Zamora-Crescencio P, Domínguez-Carrasco M, Villegas P, Gutiérrez-Báez C, Manzanero-Acevedo LA, OrtegaHaas JJ, Hernández-Mundo S, Puc-Garrido EC, PuchChávez R. 2011. Composición florística y estructura de la vegetación secundaria en el norte del estado de Campeche, México. Boletín de la Sociedad Botánica de México 89: 27-35. DOI: http://dx.doi.org/10.17129/ $\underline{\text { botsci. } 368}$ 